\title{
Length of remdesivir treatment in patients with severe COVID-19
}

\author{
Commentary on: \\ Goldman JD, et al. Remdesivir for 5 or 10 Days \\ in Patients with Severe Covid-19. N EnglJ Med \\ 2020; 383: 1827-1837.
}

\section{Context}

Coronavirus disease 2019 (COVID-19), caused by severe acute respiratory syndrome coronavirus 2 (SARS-CoV-2) infection, has caused more than 96 million cases and over 2 million deaths worldwide as of January 21, 2021. As the crisis continues, the scientific community, institutions and pharmaceutical industries are striving to find effective therapies to prevent or treat the disease. Remdesivir (GS-5734) was early identified as an option.

Remdesivir is an inhibitor of viral RNA polymerase. It had previously showed efficacy against SARS-CoV-1 and MERS-CoV in vitro. To date, three randomised controlled trials [1-3] have been published on its use for patients with COVID-19. Data from the remdesivir arm of the World Health Organization (WHO) Solidarity trial were also made available [4].

We provide a commentary on the trial by Goldman et al. [3], recently published in the New England Journal of Medicine (NEJM) comparing a 5- versus 10-day course of treatment with remdesivir in severe COVID-19 patients.

\section{Methods}

The "Study to Evaluate the Safety and Antiviral Activity of Remdesivir (GS-5734) in Participants With Severe Coronavirus Disease (COVID-19)" was a multicentre, open-label, company-funded, randomised phase III clinical trial on the use of remdesivir for patients with severe COVID-19, defined as patients with confirmed SARS-COV-2 infection within the 4 days preceding randomisation, radiographical evidence of pulmonary involvement and receiving supplemental oxygen or having oxygen saturation below $94 \%$ at room air [3]. Exclusion criteria were age $<12$ years, mechanical ventilation, use of extracorporeal membrane oxygenation (ECMO), multiorgan failure, liver enzymes levels greater than 5-times the normal range, estimated creatinine clearance of $<50 \mathrm{ml} \mathrm{min}^{-1}$ and concurrent experimental treatment for COVID-19.

The patients were randomised to receive remdesivir for a total of 5 days or 10 days, with a therapeutical scheme of $200 \mathrm{mg}$ intravenous remdesivir on day 1 , followed by $100 \mathrm{mg}$ for the subsequent days, same route. A total of 55 centres were involved, distributed among eight countries (USA, Italy, Spain, Germany, Hong Kong, Singapore, South Korea and Taiwan) from 6 to 26 March 2020.
Cite as: Ippolito M, Cortegian A. Length of remdesivir treatment in patients with severe COVID-19. Breathe 2021; 17: 200276.

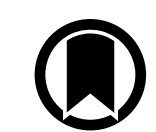

CrossMark 
The primary outcome was the score of a sevenpoint ordinal scale assessing patients' clinical status, with categories ranging from 1 , meaning death, and 7, as not hospitalised. Other efficacy outcomes were time to clinical improvement, time to recovery and time to modified recovery (defined as an improvement from a baseline score of 2 to 4 to a score of 5 to 7 or from a score of 5 to a score of 6 or 7), and death for any cause. The secondary outcome was the rate of adverse events by 30 days after the last dose.

\section{Main results}

A total of 397 patients received the treatment after randomisation (200 in the 5-day group, 197 in the 10-day group). Median (interquartile range) time of treatment was 5 (5-5) days and 9 (5-10) days in the 5- and 10-day groups respectively. The two groups were slightly unbalanced in terms of severity of disease, with the 10-day group comprising more patients starting mechanical ventilation or high-flow oxygen therapy (HFOT) before receiving the treatment. For efficacy and safety analysis, the investigators analysed all patients who were randomised and received at least one dose of remdesivir. The main results of the study are summarised in table 1.

At day 14 , clinical improvement, at least 2 points in a 7 -point ordinal scale, was registered in $64 \%$ of the patients who had received the treatment for 5 days, and in 54\% of those who had received it for 10 days. In the adjusted analysis for unbalances in baseline clinical status, patients in the 10- and 5 -day course groups had similar distribution in clinical status at day $14(p=0.14)$. The groups had similar outcomes for all the other efficacy endpoints.

The evaluation of safety outcome showed $74 \%$ $(n=145)$ of patients experiencing adverse events in the 10 -day group, and $70 \%(n=141)$ in the 5 -day group. Serious adverse events were also more frequent in the longest course treatment group, compared with the shortest course treatment group $(35 \%(n=68)$ versus $21 \%(n=42))$. The most common adverse effects in both groups were nausea, acute respiratory failure, alanine/aspartate aminotransferase increase, constipation and insomnia.

A post hoc analysis was conducted to identify any subgroup of patients who may had benefit from a long treatment (10-day course). The authors reported a lower 14 -day mortality rate among those who were under mechanical ventilation or ECMO at day 5 and assigned to the 10 days treatment group versus 5 days ( $40 \%$ (10 out of 25 ) versus $17 \%$ (7 out of 41$)$ ).

\section{Commentary}

The trial failed to demonstrate any significant difference between a 5-day versus 10-day treatment course with remdesivir in patients with severe COVID-19. Patients randomised to receive the drug for 10 days had similar or worse outcomes compared to a 5-day course of treatment. After a protocol amendment, the selected primary endpoint of the study changed to clinical status, assessed using a 7-point ordinary scale. Indeed, the original primary endpoint was normalisation of temperature and oxygen saturation through day 14. Despite it is reasonable to choose an ordinary scale, as a more sensitive measurement for small improvements, it is quite atypical to change the primary outcome during the study.

The sample size of the trial was correctly calculated on the primary outcome (odds ratio for improvement of 1.75 , power $85 \%$, two-sided $\mathrm{p}=0.05$ ). However, the lack of a control group, e.g. with placebo or standard care, is an important limitation of this study, since the efficacy of the drug cannot be evaluated. The trial was openlabel, potentially introducing performance bias. The groups were also unbalanced in terms of disease severity, and this can have affected the results. Despite randomisation, the 10-day treatment group comprised more patients under mechanical ventilation or high flow oxygen therapy, in comparison with the 5-day group. Furthermore, $44 \%$ of patients in the 10 -day cohort did not complete the entire 10 -day treatment, versus $86 \%$ in the 5-day cohort. Hospital discharge was the most common reason for incomplete treatment, and it was more common in the 10-day versus 5 -day group (35\% versus $8 \%$ ). This unbalance may be one of the reasons for the different rate of side effects, including serious side effects such as kidney failure.

In a randomised controlled trial $(\mathrm{RCT})$, no baseline differences are expected between patient groups and adjustment based on baseline variables should be unnecessary. Although the authors did not find any significant difference, the use of adjusted statistical analysis in an RCT may be considered methodologically questionable.

The study was sponsored by Gilead Science (Foster City, CA), the manufacturer of the study drug, which had role in the study design, data collection, trial monitoring, data analysis and manuscript draft. However, an independent safety monitoring committee reviewed the data after study competition for the primary outcome and guided the trial flow. Finally, the post hoc analysis included patients according to their clinical status at day 5, i.e. after having received the treatment for 5 days. This further limits the validity of the post hoc analysis, as during these five days, some patients may have worsened their clinical status before entering in the analysis. Moreover, the multiplicity of the analyses poses the risk of randomly finding a positive but unreal result.

It remains difficult to say if the differences between the two treatment regimens may be related to the study limitations or drug toxicity. 


\section{Implications for practice}

The trial suggested that in case of decision to use remdesivir in severe COVID-19, a 5-day regimen may be as effective as a 10-day course in terms of clinical efficacy but safer in terms adverse events. This information should be put in context of the evidence already available on the drug. The first RCT on remdesivir, by WANG et al. [1], had previously showed no effect on any clinical or biological endpoint (including viral load). Another trial by BeIGEL et al. [2], published in the NEJM in the same date of the one by Goldman et al. [3], demonstrated a reduction of time to recovery, in comparison with placebo (median 10 days versus 15 days; rate ratio for recovery $1.29 ; 95 \% \mathrm{Cl} 1.12-1.49 ; \mathrm{p}<0.001$ ), suggesting potential benefit for healthcare systems.

The trial came out in a challenging research context, with lack of robust evidence to guide clinical recommendations and many studies published and disseminated despite methodological flaws [5-7]. This RCT may be considered a questionable allocation of resources. Testing different dosing schemes without a control group, in the absence of data demonstrating the efficacy or the safety of the drug, was probably premature. High-quality RCTs, especially those with adaptive
Table 1 Summary of the main results

\begin{tabular}{lcc}
\hline & 5-day & 10-day \\
\hline Subjects & 200 & 197 \\
Clinical status of 2-3 points at baseline & $53(26)$ & $69(35)$ \\
Clinical improvement at day 14 & $129(64)$ & $107(54)$ \\
Any adverse events & $141(70)$ & $145(74)$ \\
Any serious adverse events & $42(21)$ & $68(35)$
\end{tabular}

Data are presented as $\mathrm{n}$ or $\mathrm{n}(\%)$. The reported numbers per group represent the number of patients per outcome. Information from [3].

design, would reasonably provide conclusive data on experimental treatments for COVID-19, thus limiting overtreatment and "low-value care" [5-7].

The available results from the Solidarity Trial, coordinated by the WHO, showed that remdesivir may have little or no effect on 28-day mortality or in-hospital clinical course of hospitalised patients with COVID-19 [4]. In light of the available data, WHO issued a conditional recommendation against the use of remdesivir in hospitalised patients, regardless of disease severity. Currently, there is no evidence that remdesivir has effects on survival and other outcomes in patients with COVID-19.

\section{Affiliations}

Mariachiara Ippolito ${ }^{1}$, Andrea Cortegiani ${ }^{1,2}$

${ }^{1}$ Dept of Surgical, Oncological and Oral Science (Di.Chir.On.S.), University of Palermo, Palermo, Italy. ${ }^{2}$ Dept of Anesthesia, Intensive Care and Emergency, Policlinico Paolo Giaccone, Palermo, Italy.

\section{Conflict of interest}

M. Ippolito has nothing to disclose. A. Cortegiani has nothing to disclose.

\section{References}

1. Wang Y, Zhang D, Du G, et al. Remdesivir in adults with severe COVID-19: a randomised, double-blind, placebo-controlled, multicentre trial. Lancet 2020; 395: 1569-1578.

2. Beigel JH, Tomashek KM, Dodd LE, et al. Remdesivir for the treatment of Covid-19 - Final Report. N Engl J Med 2020; 383: 1813-1826.

3. Goldman JD, Lye DCB, Hui DS, et al. Remdesivir for 5 or 10 Days in Patients with Severe Covid-19. N Engl J Med 2020; 383: 1827-1837.

4. World Health Organization. WHO recommends against the use of remdesivir in COVID-19 patients. www.who.int/news-room/ feature-stories/detail/who-recommends-against-the-use-ofremdesivir-in-covid-19-patients Date last updated: January 19, 2021; date last accessed: January 21, 2021.

5. Mathioudakis AG, Fally M, Hashad R, et al. COVID-19 clinical trials: unraveling a methodological gordian knot. Am J Respir Crit Care Med 2020; 202: 635-637.

6 . Dobler CC. Poor quality research and clinical practice during COVID-19. Breathe 2020; 16: 200112.

7. Cortegiani A, Catalisano G, Ippolito M, et al. Retracted papers on SARS-CoV-2 and COVID-19. Br J Anaesth 2021; In press [https://doi.org/10.1016/j.bja.2021.01.008]. 